Cite as: Corominas-Roso, M., Roncero, C., Eiroa-Orosa, F. J., Gonzalvo, B., Grau-Lopez, L., Ribases, M., ... Casas, M. (2013). Brain-derived neurotrophic factor serum levels in cocaine-dependent patients during early abstinence. European Neuropsychopharmacology, 23(9), 1078-1084.

https://doi.org/10.1016/j.euroneuro.2012.08.016

\title{
Brain-derived neurotrophic factor serum levels in cocaine-dependent patients during early abstinence
}

Margarida Corominas-Roso ${ }^{1}$, Carlos Roncero ${ }^{1-4}$, Francisco Jose Eiroa-Orosa ${ }^{1,2}$, Begoña Gonzalvo ${ }^{1,3}$, Lara Grau-Lopez ${ }^{1,3}$, Marta Ribasés ${ }^{1,4,5}$, Laia Rodriguez-Cintas ${ }^{3}$, Cristina Sánchez-Mora ${ }^{1,5}$ Josep-Antoni Ramos-Quiroga ${ }^{1,4}$, and Miguel Casas ${ }^{1-4}$

${ }^{1}$ Department of Psychiatry, Hospital Universitari Vall d'Hebron, Universitat Autònoma de Barcelona, Barcelona, Catalonia, Spain

2 Department of Psychiatry and Legal Medicine, Universitat Autònoma de Barcelona, Catalonia, Spain

${ }^{3}$ Outpatient Drug Clinic Vall Hebron, Psychiatry Services, Hospital Universitari Vall d'Hebron-ASPB, Barcelona, Catalonia, Spain

4 Biomedical Network Research Center on Mental Health (CIBERSAM), Barcelona, Catalonia, Spain

${ }^{5}$ Psychiatric Genetics Unit, Vall d'Hebron Research Institute (VHIR), Barcelona, Catalonia, Spain

\section{Corresponding author:}

Dr. Margarida Corominas-Roso, Psychiatry Service, Vall d'Hebron University Hospital, Pg. Vall d'Hebron, 119-129, 08035 Barcelona, Spain.

Phone: 34-934-89-42-94; 34-934-89-42-95

Fax: $34-934-89-45-87$

E-mail:mcoromin@vhebron.net; mgtc@neuroclassics.org

Website: http://www.neuroclassics.org

Abstract: 233 words

Text: 2484 words

Figures: 3

Tables: 4 


\begin{abstract}
Preclinical studies indicate that brain-derived neurotrophic factor (BDNF) is involved in neuroplastic changes underlying enduring cocaine-seeking following withdrawal. However, little is known about temporal changes in serum BDNF levels or the involvement of BDNF in craving and abstinence in early-abstinent cocaine-dependent patients. Twenty-three cocaine-dependent individuals (aged $33.65 \pm 6.85$ years) completed a two-week detoxification program at an inpatient facility. Two serum samples were collected for each patient at baseline and at the end of the protocol. Serum samples were also collected for 46 healthy controls (aged $35.52 \pm 9.37$ years). Demographic, consumption and clinical data were recorded for all patients. Significantly lower serum BDNF levels $(\mathrm{p}<.0001)$ were observed for cocaine-dependent patients at baseline compared to healthy controls. Serum BDNF levels increased significantly across 12 days of early abstinence $(\mathrm{p}=.030)$. Baseline BDNF levels correlated with craving $(\mathrm{p}=.034)$. Post-detoxification BDNF levels correlated with craving $(\mathrm{p}=.018)$, loss of control $(\mathrm{p}<.000)$, abstinence measures $(\mathrm{p}=0.031)$, depression $(\mathrm{p}=0.036)$, and anxiety $(\mathrm{p}=0.036)$. Post-detoxification BDNF levels also had predictive value for the loss of control measure of craving. Chronic cocaine use is associated with decreased serum BDNF. A progressive increase in serum BDNF levels during early abstinence correlates with cocaine craving and abstinence symptoms and may reflect increasing BDNF levels in different brain regions. These findings suggest that serum BDNF may be a biomarker for cocaine addiction.
\end{abstract}

Key words: Abstinence; Addiction; Cocaine; Craving; Brain-Derived Neurotrophic Factor; BDNF 


\section{Introduction}

Despite cocaine abuse is a significant public health problem, an effective pharmacotherapeutic treatment is still lacking (Castells et al., 2010). Identification of candidate biomarkers could assist in the development of effective pharmacological agents.

There is now considerable evidence that neurotrophins such as brain-derived neurotrophic factor (BDNF) and its intracellular signaling pathways are involved in psychostimulant abuse and dependence (see Corominas et al., 2007 for a review). A single infusion of cocaine increases BDNF expression in the nucleus accumbens (NAc) (Filip et al., 2006) and prefrontal cortex (PFC) (Le Foll et al., 2005). Cocaine selfadministration increases BDNF expression in the rat PFC, thereby modifying the reinforcing effects of cocaine (Sadri-Vakili et al., 2010). In rodents treated repeatedly with cocaine, brain BDNF expression increases progressively after withdrawal (Grimm et al., 2003; Pu et al., 2006).These studies also suggest that the progressive increase in BDNF expression in different brain areas after withdrawal is involved in incubation of cocaine craving (Grimm et al., 2003) and depressive behavioral symptoms (Filip et al., 2006). Additionally, overexpression of BDNF in the mesolimbic system enhances locomotor sensitization and the rewarding effects of cocaine (Bahi et al., 2008). Finally, heterozygous BDNF knockout mice $(B D N F+/-)$ are less sensitive to the psychomotor stimulant and the rewarding effects of cocaine (Hall et al., 2003).

Since BDNF crosses the blood-brain barrier in both directions (Pan et al., 1998) and can be measured in serum and plasma (Fujimura et al., 2002), serum BDNF levels may reflect brain BDNF levels (Klein et al., 2011). The first study assessing BDNF in cocaine addicts found no difference in BDNF serum levels in 15 cocaine addicts and a group of healthy controls (Angelucci et al., 2007). However, a recent cross-sectional 
study of 22 cocaine-dependent individuals reported a significant correlation between serum BDNF and number of abstinence days (Hilburn et al., 2011) and recently, BDNF was reported to be increased after 3 weeks of withdrawal (D’Sa et al., 2011).

In abstinent cocaine-dependent patients, an intense desire for the drug (craving) and symptoms of physical and mental distress are strong predictors of relapse (Garavan et al., 2000). However, no controlled studies have linked these symptoms with blood BDNF levels. In the present study, we explored whether serum BDNF levels are a biomarker of cocaine craving and withdrawal.

\section{Experimental procedures}

\section{Subjects}

Patients met the diagnostic of cocaine addiction, according to the DSM-IV (American Psychiatric Association, 2000) criteria and were drawn from a cohort involved in an independent clinical study (ClinicalTrials.gov Identifier: NCT00495092). Exclusion criteria included: 1) Lifetime history of substance use disorder except nicotine; 2) lifetime history of psychotic, bipolar, or substance abuse disorders; 3) current history of mood, psychotic or anxiety disorder; 4) organic illness; 5) being currently under psychotropic medication at least one month before joining the protocol; 6) seropositive for HIV; 7) psychotic symptoms during abstinence from or consumption of cocaine; 8) patients on chronic drug therapy with corticosteroids, thyroid hormones, allergy and/or analgesics. Sixty-two cocaine-dependent patients were assessed for eligibility; of these, 23 met the diagnostic criteria for inclusion (Figure 1). A genderand age-matched sample of 46 healthy controls was also included. All participants were Caucasian and unrelated to one another. 
This study was approved by the Clinical Research Ethics Committee of the University

Hospital Vall d'Hebron. Written informed consent was obtained from all participants.

Figure 1. Flow Diagram of the study.

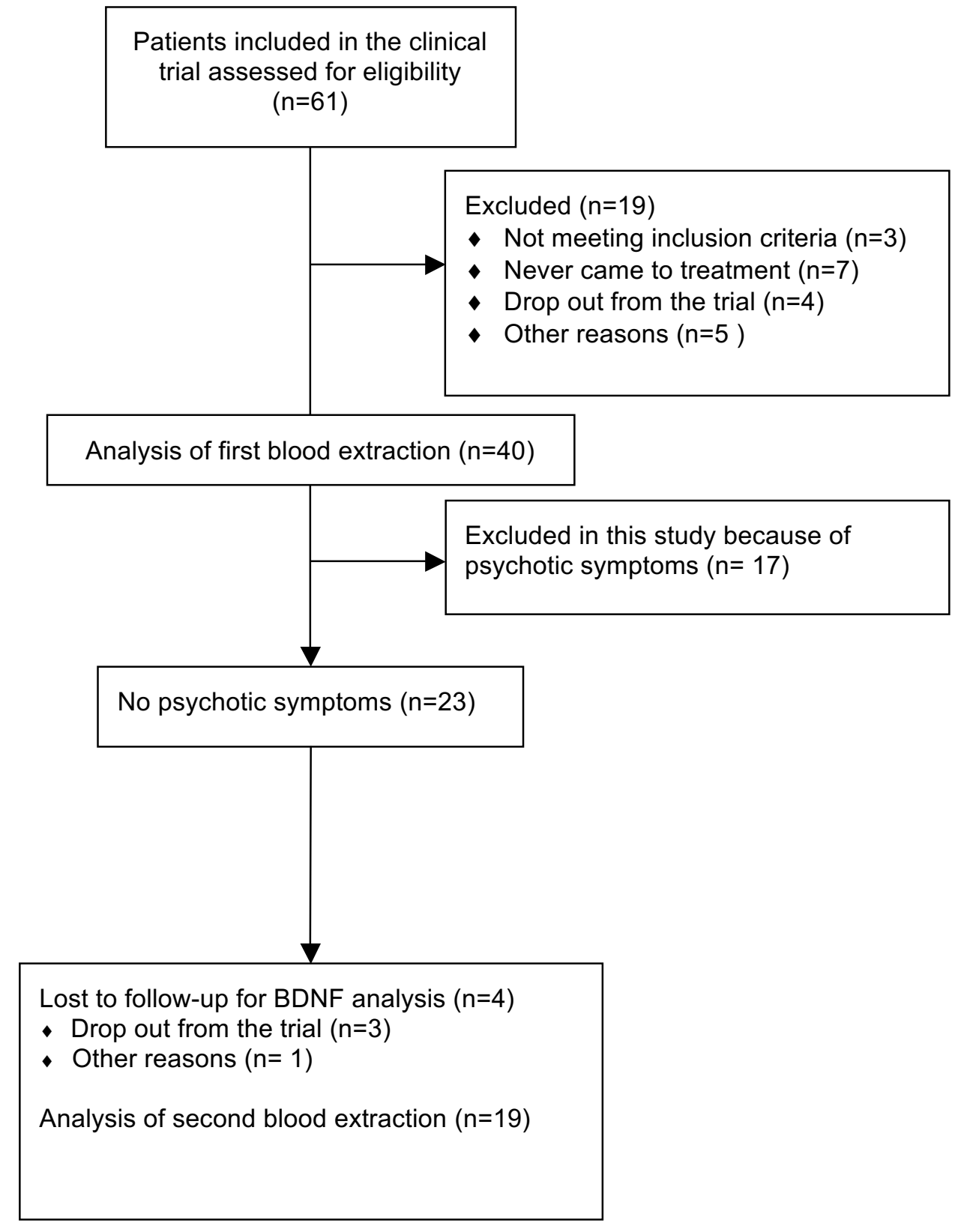




\section{Inpatient Procedure}

Two clinicians independently examined the patients and determined whether they met the criteria for inclusion. Patients were admitted to the inpatient unit of the Department of Psychiatry, Vall d'Hebron University Hospital, for 12 days. The inpatient unit is a locked treatment facility in which patients have no access to alcohol or drugs and limited visitation. Drug testing was conducted twice to ensure abstinence. Smoking was permitted during a maximum of six breaks per day previously established by the medical team.

As part of the independent clinical study, patients received caffeine (starting dose of $300 \mathrm{mg} / \mathrm{d}$; dose increased by $300 \mathrm{mg} / \mathrm{d}$; maximum dose was $15 \mathrm{mg} / \mathrm{kg} / \mathrm{d}$ up to $1200 \mathrm{mg} / \mathrm{d}$ ) and Biperiden (4 to $8 \mathrm{mg} /$ day) or matched placebo. Caffeine was administered to improve patients' comfort and Biperiden was administered to avoid tolerance to caffeine (ClinicalTrials.gov Identifier: NCT00495092). No additional psychotropic medication was administered except Lorazepam (up to $5 \mathrm{mg} / \mathrm{d}$ ) for the treatment of insomnia.

\section{Clinical Assessment}

Psychiatric diagnoses and cocaine dependence were evaluated with the Structured Clinical Interview for DSM-IV-TR (SCID) I and II. The Visual Analogic Scale (VAS) Craving for cocaine, the State Trait Anxiety Inventory, the Beck Depression Inventory, the Cocaine Craving Questionnaire (CCQ) and the Cocaine Selective Severity Assessment, were administered.

The CCQ included five dimensions of cocaine craving: a) desire, b) anticipation of positive reinforcement, c) anticipation of negative reinforcement, d) intention and planning to consume, and e) perceived lack of control over use. 


\section{Blood sample collection AND BDNF measurements}

Blood samples were collected twice, the first, immediately after arriving at the impatient facility and the second one, after twelve detoxification days. All patients had consumed cocaine the day before admission. $8 \mathrm{ml}$ of blood was collected in anticoagulant-free tubes, kept at $4^{\circ} \mathrm{C}$ for two hours, centrifuged at $3500 \times \mathrm{g}$ for $10 \mathrm{~min}$ at $4^{\circ} \mathrm{C}$ and serum stored at $-80^{\circ} \mathrm{C}$ until performance of the BDNF assay. All samples were collected at fasting state and between 10 and $12 \mathrm{~h}$ to avoid circadian variations.

Levels of human BDNF in serum samples were measured using Aushon SearchLight Multiplex Array (Aushon BioSystems, Billerica, MA) a sandwich enzymelinked immunosorbent system. Each sample was analyzed twice and the mean of the two BDNF measurements was used. All mean intra-assay coefficients of variation were less than $20 \%$.

\section{Statistical analyses}

Chi squared (for demographic categorical variables) and U Mann Whitney tests (for BDNF serum levels) were used to analyze differences between patients and controls and Wilcoxon signed-rank test for longitudinal changes in treated patients. Boxplots were additionally used to describe quartile $(\mathrm{Q} 1)$, median $(\mathrm{Q} 2)$ and upper quartile $(\mathrm{Q} 3)$ of BDNF serum levels in both samples (pre and post in the case of patients), as they didn't follow a normal distribution. Spearman's Rho was used to correlate BDNF with age, consumption variables and baseline clinical (abstinence, craving, depression, anxiety) measures. Kruskall wally's test was used to analyze the differences between the three treatment groups. A stepwise linear regression was used to assess the best clinical baseline correlates in BDNF serum change among the clinical sample. Statistical hypotheses were two-tailed and the risk alpha assumed was 5\% in all analyses. All analyses were carried out using PASW Statistics software package, version 18. 


\section{Results}

\section{Demographic characteristics and evaluation of clinical symptoms}

The clinical group consisted of 23 cocaine addicts ( 21 men and 2 women) and 46 healthy matched controls (41 men and 5 women). Comparison of basic demographic data (gender and age) of patients and controls can be seen in table 1. No statistically significant differences were found for both variables between groups. Clinical and sociodemographic data of the clinical sample can be found in table 2.

Table 1. Sociodemographic features by patient and control groups.

\begin{tabular}{lllllllc}
\hline & \multicolumn{2}{l}{ PATIENTS $(\mathrm{n}=23)$} & \multicolumn{3}{c}{ CONTROLS $(\mathrm{n}=46)$} & \multicolumn{3}{c}{ Significance } \\
\hline Gender (\% female) & $\mathrm{N}$ & $\%$ & $\mathrm{~N}$ & $\%$ & $\chi^{2}$ & $\mathrm{p}$ \\
& 2 & 8.7 & 5 & 10.9 & 0.79 & .778 \\
\hline Age & $\mathrm{M}$ & $\mathrm{SD}$ & $\mathrm{M}$ & $\mathrm{SD}$ & $\mathrm{t}$ & $\mathrm{p}$ \\
& 33.65 & 6.85 & 35.52 & & 9.37 & -.849 & .399 \\
\hline
\end{tabular}

Table 2. Clinical information of patients. Includes consumption data, craving, depression and anxiety measures at detoxification treatment baseline. VAS: Visual Analogic Scale Craving for cocaine; CCQ: Cocaine Craving Questionnaire; CSSA: Cocaine Selective Severity Assessment; BDI: Beck Depression Inventory; STAI: State Trait Anxiety Inventory.

\begin{tabular}{lll}
\hline Consumption variables & Mean & SD \\
\hline Age of onset & 23.95 & 13.65 \\
Days of consumption in the last month & 20.77 & 7.55 \\
Consumption episodes in the last month & 3.53 & 2.48 \\
Maximum quantity in 24 hours in the last month (g) & 3.31 & 4.13 \\
\hline Consumption pattern (n,\%) & 12 & \\
Daily & 7 & 52.2 \\
Regular episodes & 4 & 30.4 \\
$\quad$ Irregular episodes & & 17.4 \\
\hline Baseline ${ }^{1}$ addiction measures & 3.27 & \\
VAS (1-10) & 22.00 & 3.33 \\
CCQ $\quad 23.63$ & 10.61 \\
$\quad$ Desire & 21.19 & 9.23 \\
$\quad$ Intention & 21.44 & 9.77 \\
$\quad$ Anticipation & 42.88 & 7.79 \\
$\quad$ Relief & 13.21 & 17.03 \\
$\quad$ Loss of control & & 9.56 \\
CSSA (18 symptoms) & 12.61 & \\
BDI, (cut-off for moderate depression=7) & & 9.33 \\
\hline
\end{tabular}




\section{Serum BDNF levels in controls and patients during early cocaine abstinence}

Fourteen patients received caffeine (three of them also with biperiden) and 9 received placebo. No statistical significant differences were detected at baseline $(\mathrm{z}=$ $.189, \mathrm{p}=.877)$, post-detoxification $(\mathrm{z}=-1.352, \mathrm{p}=.196)$ or increase $(\mathrm{z}=-1.690, \mathrm{p}=.100)$ of BDNF serum levels between patients with or without caffeine treatment (for more information please see ClinicalTrials.gov Identifier: NCT00495092). Hence, all treatment groups were analyzed together.

Table 3 presents the BDNF serum levels in controls, patients at baseline, and patients after 12 days of abstinence. BDNF levels were significantly lower in patients at baseline than in controls $(\mathrm{z}=-3.488 ; \mathrm{p}<.0001)$. BDNF levels increased significantly in patients across 12 days of abstinence $(\mathrm{z}=-2.173 ; \mathrm{p}=.030)$. Analyses of normality showed that in patients, skewness and kurtosis was near 0 both at intake and after 12 days of abstinence, whereas in controls skewness was 1.6 and kurtosis was 3.6, indicating a leptokurtic distribution. Figure 2 depicts Q1, Q2, and Q3 in controls, patients at intake, and patients after 12 days of abstinence. 
Table 3. Means (M) and standard deviations (SD) of Brain Derived Neurotrophic Factor (BDNF) among healthy controls and cocaine addicted patients. Significant differences were found between patients and controls at baseline and longitudinally among patients between baseline and 12 days of abstinence (post-detoxification).

\begin{tabular}{lllllll}
\hline & PATIENTS & \multicolumn{3}{l}{ CONTROLS } & \multicolumn{2}{l}{ Significance } \\
\hline & $\mathrm{M}$ & $\mathrm{SD}$ & $\mathrm{M}$ & $\mathrm{SD}$ & $\mathrm{z}$ & $\mathrm{p}$ \\
\hline BDNF baseline & 51.676 & 17.505 & 76.044 & 32.661 & -3.488 & $<.0001$ \\
$\begin{array}{l}\text { BDNF post } \\
\text { detoxification }\end{array}$ & 60.643 & 22.607 & 76.044 & 32.661 & -1.630 & .103 \\
Significance & $\mathrm{z}=-2.173$ & $\mathrm{p}=.030$ & & & & \\
\hline
\end{tabular}

Figure 2. Boxplot of BDNF serum levels. Serum brain-derived neurotrophic factor (BDNF) levels ( $\mathrm{ng} / \mathrm{mL}$ median) obtained from the control group (left; $\mathrm{n}=46$ ), for the patients group at baseline (middle, patients pre, $n=23$ ) and after 12 days of early detoxification treatment (right, patients post, $n=23$ ). Statistical sig- nificant differences in BDNF serum levels were found (1) at baseline between controls and patients and (2) across 12 days of abstinence between baseline and post-early detoxification

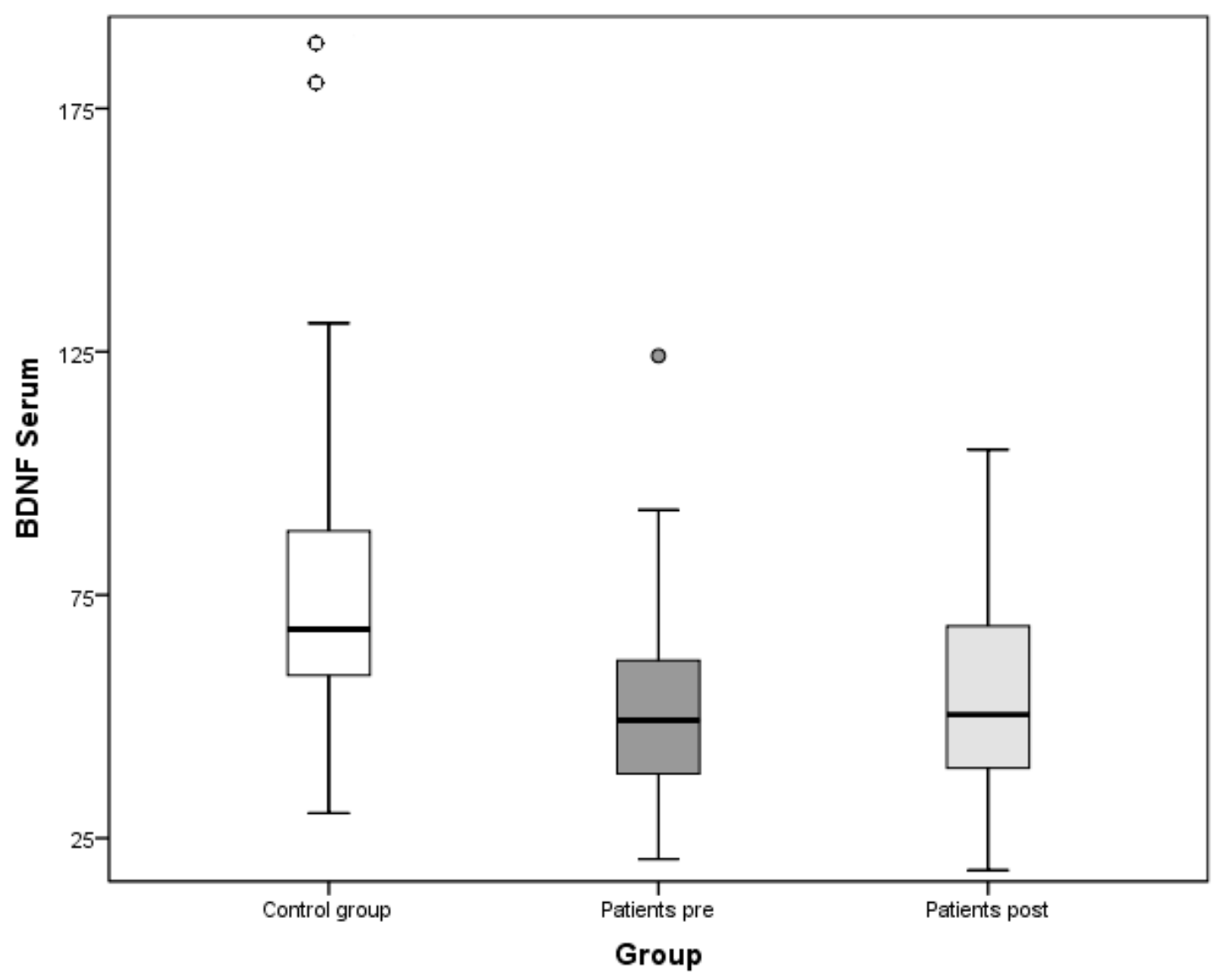




\section{Correlations between BDNF levels and demographic factors and clinical symptoms}

No correlation was found between BDNF levels and age among patients or controls. We were unable to assess correlations of BDNF levels with gender due to the small number of females in our sample. No differences were found between pharmacological treatment groups hence, we pooled the patient data for these analyses.

Of the clinical symptom measures, only craving VAS correlated significantly with baseline BDNF serum levels in patients. By contrast, post-detoxification BDNF levels correlated with craving VAS, CCQ loss of control, CSSA, BDI, and STAI trait. The increase in BDNF levels from intake to post-detoxification correlated with craving VAS, CCQ loss of control, CSSA, and BDI (Table 4). 
Table 4. Non parametric correlations and significance levels of serum Brain Derived Neurotrophic Factor (BDNF) with clinical and severity variables. Includes BDNF measures at detoxification treatment baseline (BDNF baseline), measures of BDNF after 12 days of treatment (BDNF post detoxification) and BDNF differences between baseline and post detoxification (BDNF difference). VAS: Visual Analogic Scale Craving for cocaine; CCQ: Cocaine Craving Questionnaire; CSSA: Cocaine Selective Severity Assessment; BDI: Beck Depression Inventory; STAI: State Trait Anxiety Inventory.

\begin{tabular}{|c|c|c|c|c|c|c|}
\hline & \multicolumn{2}{|c|}{ BDNF baseine } & \multirow{2}{*}{$\begin{array}{l}\text { BDNF post } \\
\text { detoxification } \\
\text { Correlation }\end{array}$} & \multirow[b]{2}{*}{ Significance } & \multicolumn{2}{|c|}{ BDNF difference } \\
\hline & Correlation & Significance & & & Correlation & Significance \\
\hline \multicolumn{7}{|l|}{ Consumption variables } \\
\hline Age of onset & -.135 & .560 & .022 & .933 & .124 & .635 \\
\hline Consumption in the last month & .216 & .422 & .364 & .201 & .335 & .241 \\
\hline \multicolumn{7}{|l|}{ Baseline addiction measures } \\
\hline VAS & .453 & .034 & .550 & .018 & .558 & .016 \\
\hline \multicolumn{7}{|l|}{$\mathbf{C C Q}$} \\
\hline Desire & .086 & .760 & .216 & .478 & .427 & .146 \\
\hline Intention & -.125 & .644 & .215 & .481 & .407 & .167 \\
\hline Anticipation & .043 & .875 & .086 & .781 & .014 & .964 \\
\hline Relief & .010 & .970 & -.148 & .629 & .044 & .887 \\
\hline Loss of control & .207 & .442 & .859 & .000 & .723 & .005 \\
\hline CSSA & .161 & .583 & .647 & .031 & .697 & .017 \\
\hline \multicolumn{7}{|l|}{ Baseline clinical measures } \\
\hline BDI & .030 & .906 & .543 & .036 & .570 & .027 \\
\hline STAI, trait & .110 & .684 & .583 & .036 & .457 & .117 \\
\hline STAI, state & .122 & .652 & .327 & .275 & .382 & .197 \\
\hline
\end{tabular}


We carried out a stepwise regression to identify the baseline measure that was most predictive of the change in BDNF levels in patients. The loss of control subscale of the CCQ was the only variable that remained in the model $(\beta=.840 ; p<.005)$. The model was statistically significant $(\mathrm{F}=16.81 ; \mathrm{p}<.005)$ and the percentage of explained variance was $71 \%(\mathrm{R}$-squared $=.706)$. Figure 3 shows a scatterplot comparing the change in BDNF levels to baseline loss of control CCQ scores, along with a regression line.

Figure 3. Scatter plot and regression line of BDNF serum level change (difference between baseline and post-early detoxifica- tion) and baseline loss of control Cocaine Craving Questionnaire (CCQ) scores. Statistically significant correlations were observed between change in BDNF levels and baseline CCQ loss of control.

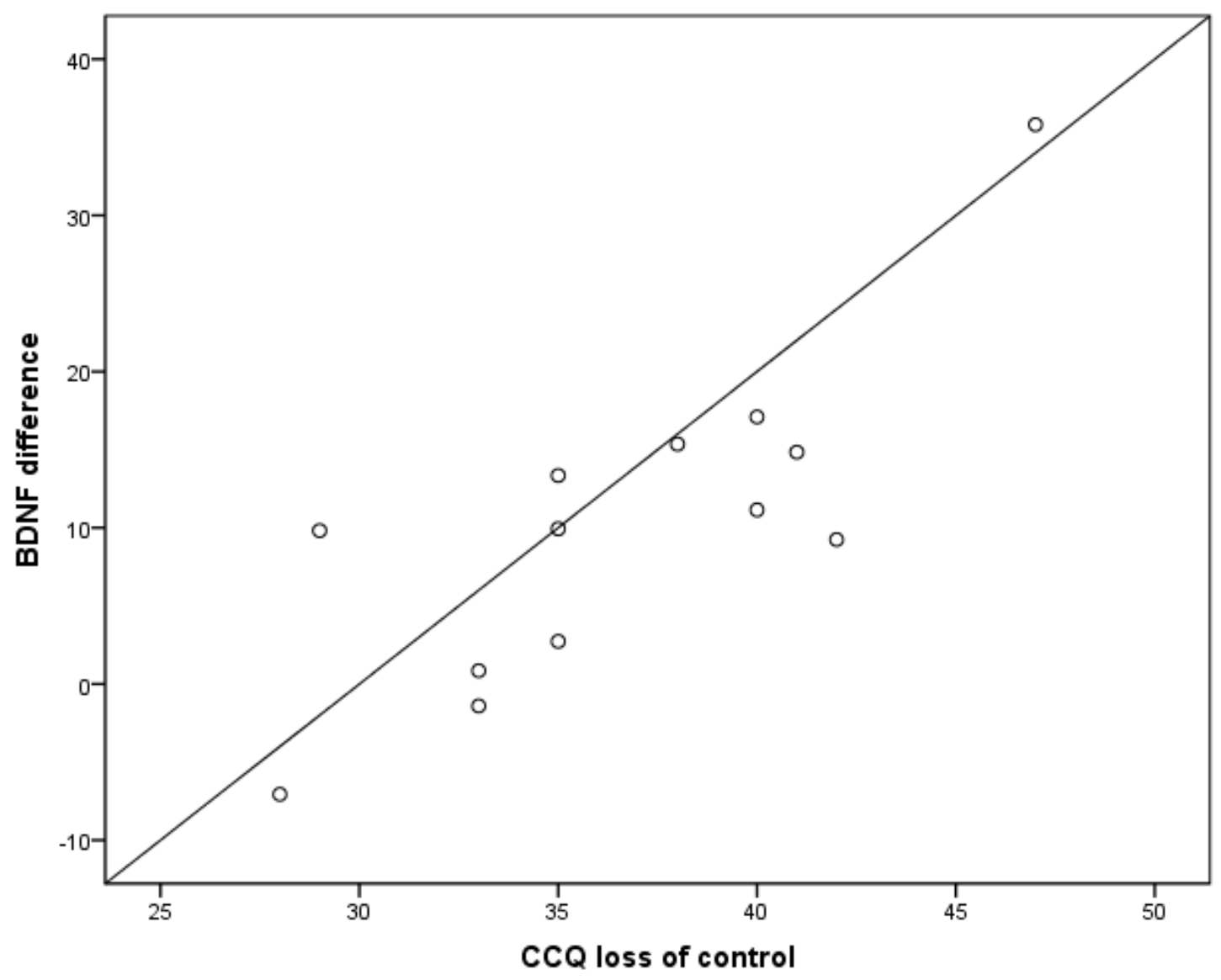




\section{Discussion}

Due to the small sample size, the results of this study have to be considered as preliminary and future studies involving larger samples will be needed. In interpreting these results, it is also necessary to take into account that about half of the patients received oral caffeine during detoxification. Although BDNF is increased after caffeine administration to zebrafish whose embryos were previously treated with caffeine (Capiotti et al., 2011) or in caffeine-treated sleep-deprived rats (Alhaider et al., 2011), we did not find differences in BDNF serum levels between caffeine- and placebotreated abstinent cocaine addicts. This may be due to the predominance of cocaine withdrawal over the stimulant effects of caffeine, an antagonist of adenosine receptors, during the first two abstinence weeks. Hence, for further comparisons in this study, data from all treatment groups were pooled and analyzed together.

Our results show a decrease of serum BDNF levels in cocaine dependent individuals, suggesting neurotoxic effects induced by chronic cocaine consumption. We also observed a significant increase in BDNF serum levels in cocaine-dependent individuals during early abstinence with the magnitude of the increase being predictive of craving. The low levels of serum BDNF in cocaine-dependent patients compared to healthy controls was somewhat unexpected, as preclinical studies in rodents report an increase in BDNF protein expression in different brain regions following acute cocaine (Le Foll et al., 2005; Liu et al., 2006). There are several possible explanations for this apparent inconsistency. First, chronic cocaine administration may have different effects than acute cocaine administration. Preclinical studies report no change in BDNF protein levels in the first days after withdrawal from chronic cocaine (Grimm et al., 2003; Pu et al., 2006) and clinical studies with human addicts show no differences in BDNF serum levels between addicts and healthy controls (Angelucci et al., 2007). 
Second, the effects of chronic cocaine consumption and withdrawal in humans differ in several respects from those seen in animal models. For example, stress vulnerability, anxiety, and depression are associated with cocaine abuse in humans (Herrero et al., 2008) and this may explain low BDNF levels reported in this study. Indeed, in our sample of cocaine-dependent patients, baseline anxiety and depression were moderately high (Table 2). Chronic stress prevents cocaine-induced BDNF upregulation in the PFC (Fumagalli et al., 2009), and low serum BDNF has been consistently reported as a biomarker for depressive-like states (Schmidt and Duman, 2010). Additionally, cocaine dependence is associated with dysregulation of the hypothalamic-pituitary-adrenal axis and increased cortisol (Fox et al., 2009) which in turn is associated with a decrease in BDNF expression (Fanous et al., 2010). According to the role of BDNF in the regulation of neuronal connectivity, synaptic plasticity and survival of dopamine neurons (Thoenen, 1995; Fritsch et al., 2011), low BDNF serum levels in cocaine-dependent patients may induce neuronal toxicity and thereby compromise synaptic plasticity in mesolimbic regions.

Our findings show a significant increase in serum BDNF levels over the first 12 days of abstinence (table 3) that appears to be independent of the caffeine treatment. Serum BDNF increased in patients being in an inpatient facility with locked doors and small and controlled environmental space that may have prevented a greater increase of BDNF during withdrawal treatment. This reported increase is consistent with preclinical models in which BDNF increases progressively in the brain (Pu et al., 2006; SadriVakili et al., 2010) up to 90 days of abstinence (Grimm et al., 2003). Our results are also in accordance with D'sa et al. (2011) who reported higher BDNF serum levels in a sample of cocaine-dependents after three abstinence weeks as compared to healthy controls. These changes in serum BDNF during early cocaine abstinence may be due to 
compensatory mechanisms to the neurotoxic effects of chronic cocaine (Sadri-Vakili et al., 2010).

One of the main findings of our study is the significant positive relationship between the increase in serum BDNF levels over early abstinence and cocaine craving. This finding is consistent with previous research. In rodent cocaine self-administration models, there is a positive relationship between the increase of BDNF in brain regions and cue-induced drug-seeking after cocaine withdrawal (Grimm et al., 2003). In addicts, serum BDNF correlates with the time of cocaine relapse (D'sa et al., 2011). We also found a positive relationship between symptoms of abstinence, anxiety, and depression and increasing serum BDNF levels during early abstinence. In neuroimaging, cueelicited cocaine craving in addicts is associated with activation of mesocorticolimbic areas (Garavan et al., 2000). Given the role of BDNF in the regulation of neuronal connectivity and synaptic plasticity (Thoenen, 1995; Fritsch et al., 2011), our data suggest functional changes in mesolimbic neuronal networks during cocaine abstinence which may underlie abnormal brain activation and emotional and behavioral correlates of cocaine craving. Together these data suggest that serum BDNF levels may be a biomarker of neuroplastic changes that lead to loss of control over consumption and to relapse.

In conclusion, chronic cocaine use is associated with decreased baseline serum BDNF levels and serum BDNF levels rise during early abstinence. The progressive increase in serum BDNF levels during early withdrawal may reflect rising BDNF levels in brain regions involved in cocaine craving. Furthermore, these changes in BDNF expression may affect synaptic plasticity in regions of the mesolimbic system that underlie craving and contribute to addiction. Taken together, these findings suggest that 
serum BDNF levels may be a biomarker in cocaine addiction. Pharmacological agents that interact with BDNF may have clinical utility in the treatment of cocaine addiction.

\section{References}

Alhaider, I.A., Aleisa, A.M., Tran, T.T., Alzoubi, K.H., Alkadhi, K.A., 2010. Chronic caffeine treatment prevents sleep deprivation-induced impairment of cognitive function and synaptic plasticity. Sleep 33, 437-44.

American Psychiatric Association, 2000. Diagnostic and statistical manual of mental disorders: DSM-IV-TR. American Psychiatric Publishing.

Angelucci, F., Ricci, V., Pomponi, M., Conte, G., Mathé, A. a, Attilio Tonali, P., Bria, P., 2007. Chronic heroin and cocaine abuse is associated with decreased serum concentrations of the nerve growth factor and brain-derived neurotrophic factor. $\mathrm{J}$ Psychopharmacol 21, 820-5.

Bahi, A., Boyer, F., Chandrasekar, V., Dreyer, J.-L., 2008. Role of accumbens BDNF and TrkB in cocaine-induced psychomotor sensitization, conditioned-place preference, and reinstatement in rats. Psychopharmacology (Berl) 199, 169-82.

Capiotti, K.M., Menezes, F.P., Nazario, L.R., Pohlmann, J.B., de Oliveira, G.M., Fazenda, L., Bogo, M.R., Bonan, C.D., Da Silva, R.S., 2011. Early exposure to caffeine affects gene expression of adenosine receptors,DARPP-32 and BDNF without affecting sensibility and morphology of developing zebrafish (Danio rerio). Neurotoxicol Teratol 33, 680-5.

Blacktop, J.M., Seubert, C., Baker, D. a., Ferda, N., Lee, G., Graf, E.N., Mantsch, J.R., 2011. Augmented Cocaine Seeking in Response to Stress or CRF Delivered into the Ventral Tegmental Area Following Long-Access Self-Administration Is Mediated by CRF Receptor Type 1 But Not CRF Receptor Type 2. Journal of Neuroscience 31, 11396-11403.

Castells, X., Casas, M., Pérez-Mañá, C., Roncero, C., Vidal, X., Capellà, D., 2010. Efficacy of psychostimulant drugs for cocaine dependence. Cochrane Database Syst Rev CD007380. 
Childress, A.R., Mozley, P.D., McElgin, W., Fitzgerald, J., Reivich, M., O’Brien, C., 1999. Limbic activation during cue-induced cocaine craving. Am J Psychiatry 156, $11-8$.

Corominas, M., Roncero, C., Ribases, M., Castells, X., Casas, M., 2007. Brain-derived neurotrophic factor and its intracellular signaling pathways in cocaine addiction. Neuropsychobiology 55, 2-13.

D’Sa, C., Fox, H.C., Hong, A.K., Dileone, R.J., Sinha, R., 2011. Increased Serum Brain-Derived Neurotrophic Factor Is Predictive of Cocaine Relapse Outcomes: A Prospective Study. Biol Psychiatry 70, $706-711$.

Filip, M., Faron-Górecka, A., Kuśmider, M., Gołda, A., Frankowska, M., DziedzickaWasylewska, M., 2006. Alterations in BDNF and trkB mRNAs following acute or sensitizing cocaine treatments and withdrawal. Brain Res 1071, 218-25.

Le Foll, B., Diaz, J., Sokoloff, P., 2005. A single cocaine exposure increases BDNF and D3 receptor expression: implications for drug-conditioning. Neuroreport 16, 1758.

Fox, H.C., Jackson, E.D., Sinha, R., 2009. Elevated cortisol and learning and memory deficits in cocaine dependent individuals: relationship to relapse outcomes. Psychoneuroendocrinology 34, 1198-207.

Fritsch, B., Reis, J., Martinowich, K., Schambra, H.M., Ji, Y., Cohen, L.G., Lu, B., 2011. Direct current stimulation promotes BDNF-dependent synaptic plasticity: Potential implications for motor learning. Neuron 66, 198-204.

Fujimura, H., Altar, C.A., Chen, R., Nakamura, T., Nakahashi, T., Kambayashi, J., Sun, B., Tandon, N.N., 2002. Brain-derived neurotrophic factor is stored in human platelets and released by agonist stimulation. Thromb Haemost 87, 728-34.

Fumagalli, F., Caffino, L., Racagni, G., Riva, M.A., 2009. Repeated stress prevents cocaine-induced activation of BDNF signaling in rat prefrontal cortex. Eur Neuropsychopharmacol 19, 402-8. 
Garavan, H., Pankiewicz, J., Bloom, a, Cho, J.K., Sperry, L., Ross, T.J., Salmeron, B.J., Risinger, R., Kelley, D., Stein, E. a, 2000. Cue-induced cocaine craving: neuroanatomical specificity for drug users and drug stimuli. Am J Psychiatry 157, 1789-98.

Goldstein, R.Z., Volkow, N.D., 2002. Reviews and Overviews Drug Addiction and Its Underlying Neurobiological Basis : Neuroimaging Evidence for the Involvement of the Frontal Cortex. Psychiatry: Interpersonal and Biological Processes 16421652.

Grimm, J.W., Lu, L., Hayashi, T., Hope, B.T., Su, T.-P., Shaham, Y., 2003. Timedependent increases in brain-derived neurotrophic factor protein levels within the mesolimbic dopamine system after withdrawal from cocaine: implications for incubation of cocaine craving. J Neurosci 23, 742-7.

Hall, F.S., Drgonova, J., Goeb, M., Uhl, G.R., 2003. Reduced behavioral effects of cocaine in heterozygous brain-derived neurotrophic factor (BDNF) knockout mice. Neuropsychopharmacology 28, 1485-90.

Herrero, M.J., Domingo-Salvany, A., Torrens, M., Brugal, M.T., 2008. Psychiatric comorbidity in young cocaine users: induced versus independent disorders. Addiction 103, 284-93.

Hilburn, C., Nejtek, V. a, Underwood, W. a, Singh, M., Patel, G., Gangwani, P., Forster, M.J., 2011. Is serum brain-derived neurotrophic factor related to craving for or use of alcohol, cocaine, or methamphetamine? Neuropsychiatr Dis Treat 7, 357-64.

Karege, F., Bondolfi, G., Gervasoni, N., Schwald, M., Aubry, J.-M., Bertschy, G., 2005. Low brain-derived neurotrophic factor (BDNF) levels in serum of depressed patients probably results from lowered platelet BDNF release unrelated to platelet reactivity. Biol Psychiatry 57, 1068-72.

Karege, F., Schwald, M., Cisse, M., 2002. Postnatal developmental profile of brainderived neurotrophic factor in rat brain and platelets. Neurosci Lett 328, 261-4. 
Klein, A.B., Williamson, R., Santini, M.A., Clemmensen, C., Ettrup, A., Rios, M., Knudsen, G.M., Aznar, S., 2011. Blood BDNF concentrations reflect brain-tissue BDNF levels across species. Int J Neuropsychopharmacol 14, 347-53.

Lepsch, L.B., Gonzalo, L.A., Magro, F.J.B., Delucia, R., Scavone, C., Planeta, C.S., 2005. Exposure to chronic stress increases the locomotor response to cocaine and the basal levels of corticosterone in adolescent rats. Addict Biol 10, 251-6.

de Lima, M.S., de Oliveira Soares, B.G., Reisser, A.A.P., Farrell, M., 2002. Pharmacological treatment of cocaine dependence: a systematic review. Addiction 97, 931-49.

Liu, Q.-R., Lu, L., Zhu, X.-G., Gong, J.-P., Shaham, Y., Uhl, G.R., 2006. Rodent BDNF genes, novel promoters, novel splice variants, and regulation by cocaine. Brain Res $1067,1-12$.

Lu, B., Chow, A., 1999. Neurotrophins and hippocampal synaptic transmission and plasticity. J Neurosci Res 58, 76-87.

Pan, W., Banks, W.A., Fasold, M.B., Bluth, J., Kastin, A.J., 1998. Transport of brainderived neurotrophic factor across the blood-brain barrier. Neuropharmacology 37 , $1553-61$.

Pu, L., Liu, Q., Poo, M., 2006. BDNF-dependent synaptic sensitization in midbrain dopamine neurons after cocaine withdrawal. Nat Neurosci 9, 605-7.

Sadri-Vakili, G., Kumaresan, V., Schmidt, H.D., Famous, K.R., Chawla, P., Vassoler, F.M., Overland, R.P., Xia, E., Bass, C.E., Terwilliger, E.F., Pierce, R.C., Cha, J.H.J., 2010. Cocaine-induced chromatin remodeling increases brain-derived neurotrophic factor transcription in the rat medial prefrontal cortex, which alters the reinforcing efficacy of cocaine. J Neurosci 30, 11735-44.

Sakata, K., Woo, N.H., Martinowich, K., Greene, J.S., Schloesser, R.J., Shen, L., Lu, B., 2009. Critical role of promoter IV-driven BDNF transcription in GABAergic transmission and synaptic plasticity in the prefrontal cortex. Proc Natl Acad Sci U S A 106, 5942-7. 
Sartorius, A., Hellweg, R., Litzke, J., Vogt, M., Dormann, C., Vollmayr, B., DankerHopfe, H., Gass, P., 2009. Correlations and discrepancies between serum and brain tissue levels of neurotrophins after electroconvulsive treatment in rats. Pharmacopsychiatry 42, 270-6.

Satomura, E., Baba, H., Nakano, Y., Maeshima, H., Suzuki, T., Arai, H., 2011. Correlations between brain-derived neurotrophic factor and clinical symptoms in medicated patients with major depression. J Affect Disord 135, 332-335.

Thoenen, H., 1995. Neurotrophins and neuronal plasticity. Science 270, 593-8.

Wolkowitz, O.M., Wolf, J., Shelly, W., Rosser, R., Burke, H., Lerner, G.K., Reus, V.I., Nelson, J.C., Epel, E.S., Mellon, S.H., 2011. Serum BDNF levels before treatment predict SSRI response in depression. Prog Neuropsychopharmacol Biol Psychiatry $35,1623-1630$. 